\title{
EXISTENCE OF WEAK SOLUTIONS \\ FOR THE NAVIER-STOKES EQUATIONS WITH INITIAL DATA IN $L^{p}$
}

\author{
CALIXTO P. CALDERÓN
}

\begin{abstract}
The existence of weak solutions for the Navier-Stokes equations for the infinite cylinder with initial data in $L^{p}$ is considered in this paper. We study the case of initial data in $L^{p}\left(R^{n}\right), 2<p<n$, and $n=3,4$. An existence theorem is proved covering these important cases and therefore, the "gap" between the Hopf-Leray theory $(p=2)$ and that of Fabes-Jones-Riviere $(p>n)$ is bridged. The existence theorem gives a new method of constructing global solutions. The cases $p=n$ are treated at the end of the paper.
\end{abstract}

\section{INTRODUCTION}

I consider in this paper the initial value problem for the Navier-Stokes equations in the infinite cylinder $S_{T}=R^{n} \times[0, T)$. Given

$$
f(x)=\left(f_{1}(x), f_{2}(x), \ldots, f_{n}(x)\right),
$$

satisfying in the distributions sense $\operatorname{div} f=0, x \in R^{n}$, we seek a solution vector $u(x, t)=\left(u_{1}(x, t), \ldots, u_{n}(x, t)\right)$ and a pressure function $P(x, t)$ such that

$$
\begin{gathered}
D_{t} u_{i}-\sum_{j=1}^{n} D_{j j} u_{i}+\sum_{j=1}^{n} u_{j} D_{j} u_{i}+D_{i} P=0, \quad(x, t) \in S_{T}, \\
\sum_{j} D_{j} u_{j}=0, \quad(x, t) \in S_{T}, \\
u(x, 0)=f(x) .
\end{gathered}
$$

Here, $D_{j}$ and $D_{t}$ denote respectively, the distributional derivatives with respect to $x_{j}$ and $t, D_{i j}$ denotes the second order derivative with respect to $x_{i}, x_{j}$; likewise, $L(u)$ will denote the heat operator applied to $u$, and $\operatorname{grad} u$, the square matrix $D_{j} u_{i}$. The first equation of $(0.1)$ takes the form

$$
L(u)+(\operatorname{grad} u)(u)+\operatorname{grad} P=0 .
$$

Following [3], I consider the functional spaces $L^{p, q}\left(S_{T}\right)$ consisting of the Lebesgue-measurable functions $u$, such that

$$
\|u\|_{p, q}(T)<\infty .
$$

Received by the editors May 31, 1988.

1980 Mathematics Subject Classification (1985 Revision). Primary 35Q10, 35K55, 35K15. 
Let us define $U_{p}(t)$ as

$$
\left.\left.\left|\int_{R^{n}}\right| u\right|^{p} d x\right|^{1 / p}
$$

Then, the mixed norm (0.3) can be written as

$$
\left.\left\|U_{p}\right\|_{q}(T) \quad \text { (usual } L^{q} \text {-norm over the interval }(0, T)\right) \text {. }
$$

The norms associated with the maximal operator $u^{*}=\sup _{t}|u|$ (the supremum is taken over $t>0$ ) are

$$
\left\|u^{*}\right\|_{p}(T)=\left(\int\left(\sup _{0<t<T}|u|\right)^{p} d x\right)^{1 / p} .
$$

J. Leray in [8] proved existence of weak solutions for all time $t>0$, for the initial values problem $(0.1)$ when $f$ is square summable and $n=3$. Furthermore, he obtained for $u$ the estimate

$$
\left(U_{2}(t)\right)^{2}+2\left(\|\operatorname{grad} u\|_{2,2}(t)\right)^{2} \leq 2\|f\|_{2}^{2} .
$$

This result was later extended to the case $n>3$ by E. Hopf in [6].

E. B. Fabes, B. F. Jones and N. M. Riviere studied in [3] the existence and uniqueness of weak solutions in the classes $L^{p, q}\left(S_{T}\right)$ for the initial values problem $(0.1)$ with data $f$ in $L^{p}$. Fabes, Jones and Riviere imposed on $p$ and $q$ the restrictions $p>n, n / p+2 / q \leq 1$, and obtained in those cases existence and uniqueness for small time $T>0$, which is a local result.

The aim of this paper is to bridge as far as possible the "gap" between the Hopf-Leray theory, $p=2$, and that of Fabes-Jones-Riviere, $p>n$.

I consider here the cases $n=3$ and $n=4$ for the initial values problem with data $f$ in $L^{p}, 2<p<n$. The case $p=n$ will be treated independently in the final part of this paper.

\section{MAIN RESUltS}

Weak solutions [3]. A function $u(x, t)$ is said to be a weak solution of the Navier-Stokes equations with inital data $f, \operatorname{div} f=0$ in the distributions sense), if for any $C^{\infty}$, rapidly decreasing vector function $v(x, t)=\left(v_{1}(x, t)\right.$, $\left.\ldots, v_{n}(x, t)\right)$, defined on $R^{n+1}(x, t)$, such that $\operatorname{div} v=0, v(x, t)=0, t>T$, we have

(a) $u \in L^{p, q}\left(S_{T}\right)$ with $p, q \geq 2$,

(b) $\int_{0}^{T} \int_{R^{n}}\left\langle u, L^{*}(v)+(\operatorname{grad} v)(u)\right\rangle d x d t=-\int_{R^{n}}\langle f(x), v(x, 0)\rangle d x$,

(c) $\operatorname{div} u(x, t)=0$ (in the distributions sense) for a.e. $t$, such that $0<t<$ $T$, where $L^{*}$ is obviously the adjoint heat operator.

Theorem A. Suppose that $f(x)$, the initial data, is a vector function, such that $f \in L^{p}\left(R^{n}\right), n=3,4,2<p<n, \operatorname{div} f=0$ (in the distributions sense). Then 
there exists a weak solution $u(x, t)$ to problem (0.1) for all time $t$, such that $0<t<T$, where $T$ can be chosen arbitrarily large, that satisfies

(i) $\|u\|_{p, 2}\left(S_{T}\right) \leq C\left(\varepsilon_{0}\right) T^{1 / 2}\left(s^{1-\beta p / n}\|f\|_{p}^{\beta p / n}+\left\|f^{s}\right\|_{p}\right)+C_{2} s^{1-p / 2}\|f\|_{p}^{p / 2}$ provided that $s>0$ is chosen so small that

$$
T^{(1-\beta) / 2}\left(s^{1-\beta p / n}\|f\|_{p}^{\beta p / n}+\left\|f^{s}\right\|_{p}\right)<\varepsilon_{0} .
$$

Here, $f^{s}$ stands for a function equal to $f$ if $|f| \leq s$ and zero otherwise, $\beta$ is fuxed, $0<\beta<1, \varepsilon_{0}$ is a fixed small quantity, $C\left(\varepsilon_{0}\right)$ depends on $\varepsilon_{0}$, and $C_{2}$ is an independent constant.

(ii) The solution constructed in (i) can be extended to all time $t>0$ in such a manner that

$$
\|u\|_{p, 2}\left(S_{T}\right)<\infty \text { for all } T>0
$$

II. Fundamental solutions, SPlitting the Data AND the equations.

THE BILINEAR FORMS, FIXED POINTS.

Fabes-Jones-Riviere [3] extended to dimension $n$ a formula found for the case $n=3$ by Oseen [9]. The Oseen-Fabes-Jones-Riviere formula gives a divergence free matrix fundamental solution $E_{i j}(x, t)$ for $n$-dimensional heat equation.

The matrix $E_{i j}(x, t)$ is defined in the following way:

$$
E(x, t)=\delta_{i j} W(x, t)-R_{i} R_{j} W(x, t),
$$

where $W(x, t)=(4 \pi t)^{-n / 2} \exp -|x|^{2} /(4 t)$, and $R_{j}$ is the $j$ th Riesz transform, namely

$$
R_{j}(f)=\text { p.v. } c_{j} \int\left(x_{j}-y_{j}\right)|x-y|^{-(n+1)} f(y) d y .
$$

For details see [3] and [12]. $E_{i j}(x, t)$ is symmetric and divergence free, that is

$$
\sum_{j} D_{j} E_{i j}(x, t)=0, \quad t \geq 0 .
$$

In the above formula we take classical derivatives if $t>0$, and distributional derivatives for the limit for $t$ tending to 0 .

Another important property is the following one:

$\sum_{j} \int E_{i j}(x, t) f_{j}(y-x) d x$ tends to $f_{i}(y)$ in $L^{p}, 1<p<\infty$, as $t$ tends to zero, provided that $\operatorname{div} f=0$.

A very important theorem in [3] asserts that $u(x, t)$ is a weak solution of problem (0.1) (see definition in $\S I$ ) over $S_{T}$, with $g \in L^{r}, 1 \leq r<\infty$, if and only if it is a solution of the following integral equation [3, Theorem 2.1, p. 226]:

$$
u+B(u, u)=F(x, t)
$$


$F(x, t)$ stands for the convolution on the space variables of the initial data $g(x)$ with fundamental solution $W(x, t) . B(u, v)$ is the bilinear form

$$
\int_{0}^{t} \int_{R^{n}}\langle(\operatorname{grad} E(x-y, t-s))(v(y, s)), u(y, s)\rangle d y d s .
$$

We have used here a notation consistent with $(0.2)$. In fact, $(\operatorname{grad} E)(v)$ is the matrix $\sum_{k} D_{k} E_{i j} v_{k}$, whose $i$ th row is dotted with $u$ to obtain the integrand of (2.5).

Splitting the equations and the data of (0.1). Consider the system in the unknowns $u, v, P$ and $Q$ :

$$
\begin{gathered}
L(u)+(\operatorname{grad} u)(u)+\operatorname{grad} P=0, \\
L(v)+(\operatorname{grad} v)(v)+(\operatorname{grad} v)(u)+(\operatorname{grad} u)(v)+\operatorname{grad} Q=0, \\
\operatorname{div} u=0, \quad \operatorname{div} v=0, \\
u(x, 0)=g(x), v(x, 0)=f(x), \operatorname{div} g=0, \operatorname{div} f=0 .
\end{gathered}
$$

Definition. The vector functions $u$ and $v$ are said to be weak solutions of problem (2.6) if $u+v$ and $u$ are weak solutions of $(0.1)$ for the initial data $g+f$ and $g$ respectively.

A consequence of the above definition and Theorem (2.1) in [3] (see also [8, p. 218]) is the following:

Theorem (II.1). The vector functions $u$ and $v$ belonging to $L^{p, q}\left(S_{T}\right), 2 \leq$ $p, q \leq \infty$ are weak solutions of problem (2.6) for the initial data $g$, and $f$ in $L^{r}, 1 \leq r<\infty$, if and only if they are solutions of the following integral equations:

$$
\begin{gathered}
u+B(u, u)=W * g, \\
v+B(v, v)+B(v, u)+B(u, v)=W * f .
\end{gathered}
$$

Furthermore, the pressure $P$ and pseudopressure $Q$ are given by

$$
\begin{aligned}
& P=C \sum_{i j} R_{i} R_{j}\left(u_{i} u_{j}\right), \\
& Q=C \sum_{i j}\left(R_{i} R_{j}\left(v_{i} v_{j}\right)+2 R_{i} R_{j}\left(v_{i} u_{j}\right)\right) .
\end{aligned}
$$

Here, $W * g$ and $W * f$ are the convolutions on the space variables of $g$ and $f$ with the fundamental solution $W . R_{i}$ stands for the ith Riesz transform and $C$ is a constant depending on $n$, the dimension.

Lemma (II.1). Let $f \in L^{p}\left(R^{n}\right), 2<p<n$, be a given vector function such that $\operatorname{div} f=0$ in the distributions sense. Then, for each $s>0, f$ can be written as the sum $g+h$, where $g$ and $h$ satisfy

$$
\begin{array}{rlrl}
\|g\|_{n} & \leq C s^{1-p / n}\|f\|_{p}^{p / n}, & & \operatorname{div} g=0, \\
\|h\|_{2} \leq C s^{1-p / 2}\|f\|_{p}^{p / 2}, & & \operatorname{div} h=0 .
\end{array}
$$

The constant $C$ depends only on $n$ and on $p$. 
Proof. First, let us recollect the property

$$
\lim \int_{R^{n}} \sum_{j} E_{i j}(x-y, t) f_{j}(y) d y=f_{i}(x) \quad \text { a.e. }
$$

The limit is taken as $t$ tends to 0 . It exists a.e. and in $L^{p}$-norm.

Choose now $s>0$ and partition $f_{j}$ as follows:

$$
f_{j}=f_{j}^{(1)}+f_{j}^{(2)}
$$

where $f_{j}^{(1)}$ equals $f_{j}$ if $\left|f_{j}\right|<s$ and equals zero otherwise. The functions $g$ and $h$ are defined in the following way:

$$
\begin{aligned}
& g_{i}(x)=\lim \int \sum_{j} E_{i j}(x-y, t) f_{j}^{(1)}(y) d y \quad \text { a.e., } \\
& h_{i}(x)=\lim \int \sum_{j} E_{i j}(x-y, t) f_{j}^{(2)}(y) d y \quad \text { a.e. }
\end{aligned}
$$

The limit in the above expressions exist a.e. and in the $L^{n}$ sense for $g$ and in the $L^{2}$ sense for $h$. This fact is a consequence of the definition of $E$ (a convolution in the space variables of the matrix singular integral operator $\left(\delta_{i j} I-R_{i} R_{j}\right)=K_{i j}$ with the $W(x, t)$ the fundamental solution of the heat equation [3]).

Finally, inequalities (2.7) are easy to verify for $f^{(1)}$ and $f^{(2)}$ which imply similar inequalities for $g$ and $h$ for appropriate values of $C$, on account of the continuity of the singular integral operator $K_{i j}$ in $L^{n}$ and in $L^{2}$.

For each $t>0 \quad(E) *\left(f^{(1)}\right)$ has null divergence. The same holds for the limit $g$, provided that the derivatives are taken in the distributions sense. A similar argument holds for $h$.

Estimates for the bilinear operators and fixed-point properties. In what follows, we are going to consider Banach spaces of Lebesgue measurable functions defined on $S_{T}$ for which the operator $T(u, v)=B(u, v)+l(u)+F(x, t) \quad(B(u, v)$ is bilinear and $l(u)$ is assumed to be linear) satisfies an inequality of the type

$$
\|T(u, v)\| \leq C_{1}\|u\|\|v\|+C_{2}\|u\|+\|F\| .
$$

In (2.9) above, the norm is that of the Banach space in question.

Lemma (II.2). The quadratic operator $T(u, u)$ maps the ball $\left\{\|u\| \leq s_{1}\right\}$ into itself if $s_{1}$ is the smallest root of

$$
C_{1} s^{2}+\left(C_{2}-1\right) s+\|F\|=0
$$

provided that $C_{1}, C_{2}$ and $\|F\|$ satisfy

$$
\left(1-C_{2}\right)^{2}>4 C_{1}\|F\|, \quad C_{1}>0,0 \leq C_{2}<1 .
$$


If $2 s_{1} C_{1}+C_{2}<1, T(u, u)$ is a contraction mapping in the ball of radius $s_{1}$. In particular, $T(u, u)$ is a contraction mapping in the ball of radius $s_{1}$ if $C_{1}$, $C_{2}$ and $\|F\|$ satisfy

$$
2 C_{1}\|F\|\left\{\left(1-C_{2}\right)^{2}-4 C_{1}\|F\|\right\}^{-1 / 2}+C_{2}<1 .
$$

Proof. The line $z=s$ and the parabola $z(s)=C_{1} s^{2}+C_{2} s+\|F\|$ intersect at $s_{1}$ and $s_{2}\left(0<s_{1}<s_{2}\right)$, provided that $(2.11)$ is satisfied, therefore, if $0<s \leq s_{1}$, we have $z(s) \leq s_{1}$. On account of (2.9), the same holds for $\|T(u, u)\|$, whenever $\|u\| \leq s_{1}$. Finally, inequality (2.12) is a consequence of a bound for $s_{1}$ in terms of the coefficients of $z(s)-s$, namely

$$
s_{1} \leq\|F\|\left\{\left(1-C_{2}\right)^{2}-4 C_{1}\|F\|\right\}^{-1 / 2} .
$$

\section{Proof of Theorem A}

Identities. Let us return to the notation introduced previously and restate it:

$$
\begin{aligned}
\left(a_{i j}\right)\left(b_{j}\right) & =\sum_{j} a_{i j} b_{j}, \\
(\operatorname{grad} v)(u) & =\sum_{j} D_{j} v_{i} u_{j}, \\
\langle(\operatorname{grad} v)(u), w\rangle & =\sum_{i j} D_{j} v_{i} u_{j} w_{i} .
\end{aligned}
$$

In addition, $I$ introduce the integral product

$$
\iint_{S_{t}}\langle(\operatorname{grad} v)(u), w\rangle d x d t=[(\operatorname{grad} v)(u), w] .
$$

If $u, v$, and $w$ are divergence free vector functions (null divergence in the distributions sense), such that their derivatives are Lebesgue-measurable and the products in the expressions below are Lebesgue integrable, we have

$$
[(\operatorname{grad} v)(u), w]=-[(\operatorname{grad} w)(u), v] .
$$

Likewise, if $u, v, w$, are the same as above, and $\beta(x)$ is a $C^{\infty}$ rapidly decreasing scalar function defined in $R^{n}$, we have

$$
[(\operatorname{grad} v)(u), \beta w]=-[\beta(\operatorname{grad} w)(u), v]-[(w \operatorname{grad} \beta)(u), v] .
$$

Identities (3.2) and (3.3) are easy to verify.

The approximating system. Let $\alpha(x)$ be a $C^{\infty}$ nonnegative, compact-supported function defined on $R^{n}$, having integral equal to 1 there. Likewise, $\alpha_{\varepsilon}$ will denote, as usual, $\varepsilon^{-n} \alpha\left(\varepsilon^{-1} x\right)$ and $u^{\#}(x, t, \varepsilon)$ the convolution on the space variables $\alpha_{\varepsilon} * u(x, t)$. 
Adapting an idea of Leray's, we modify system (2.6) as follows:

$$
\begin{gathered}
L(u)+(\operatorname{grad} u)\left(u^{\#}\right)+\operatorname{grad} P=0, \\
L(v)+(\operatorname{grad}\{v+u\})\left(v^{\#}\right)+(\operatorname{grad} v)\left(u^{\#}\right)+\operatorname{grad} Q=0, \\
\operatorname{div} u=0, \quad \operatorname{div} v=0, \\
u(x, 0)=g^{\#}(x), \quad v(x, 0)=h^{\#}(x) .
\end{gathered}
$$

The initial data $g(x)$ and $h(x)$ are a partitioning of the initial value $f(x)$ by using formulae (2.8) of $\S I$ for a suitably small value of $s$. Here, $f(x)$ is a divergence free vector function belonging to $L^{p}\left(R^{n}\right), 2<p<n$. As it is immediately seen, $g^{\#}$ and $h^{\#}$ are divergence free and furthermore, they satisfy the estimates

$$
\begin{aligned}
\left\|g^{\#}\right\|_{n} & \leq\|g\|_{n} \leq C s^{1-p / n}\|f\|_{p}^{p / n}, \\
\left\|h^{\#}\right\|_{2} & \leq\|h\|_{2} \leq C s^{1-p / 2}\|f\|_{p}^{p / 2} .
\end{aligned}
$$

This is a consequence of Lemma (II.1).

Calling $w=u+v$ system (3.4) gives

$$
\begin{gathered}
L(w)(\operatorname{grad} w)\left(w^{\#}\right)+\operatorname{grad}\{P+Q\}=0, \\
\operatorname{div} w=0, \quad w(x, 0)=f^{\#} .
\end{gathered}
$$

System (3.6) is the approximating system studied by Leray in [8] used to prove existence of turbulent solutions for initial data in $L^{2}$. Similar considerations as the ones of Theorem (II.1) give for the approximating pressure $P$ and the approximating pseudopressure $Q$ the expressions

$$
\begin{aligned}
& P=C \sum_{i j} R_{i} R_{j}\left(u_{i} u_{j}^{\#}\right), \\
& Q=C \sum_{i j} R_{i} R_{j}\left(v_{i} v_{j}^{\#}+v_{i} u_{j}^{\#}+u_{i} v_{j}^{\#}\right) .
\end{aligned}
$$

The expressions for $P$ and $Q$ are a consequence of Oseen's formulae applied to the approximating system (3.7).

Finding $u$ in system (3.4). The problem reduces to solving the integral equation

$$
u+B\left(u, u^{\#}\right)=G^{\#}(x, t) \text {. }
$$

In the above expression $G^{\#}$ stands for the convolution of $g^{\#}$ with the fundamental solution of the heat equation $W(x, t)$.

Lemma (III.1). The equation (3.8), including the limit case, that is when $u=u^{\#}$ and $G=G^{\#}$, admits a unique solution $u$ satisfying

$$
\left\|u^{*}\right\|_{n}(\infty) \leq C s^{1-p / n}\|f\|_{p}^{p / n}
$$

provided that $s^{1-p / n}\|f\|_{p}<\varepsilon_{0}$, 
(ii)

$$
\left\|u^{*}\right\|_{p}(\infty) \leq C \max \left(s^{1-p / n}\|f\|_{p}^{p / n},\left\|f^{s}\right\|_{p}\right)
$$

provided that $\max \left(s^{1-p / n}\|f\|_{p}^{p / n},\left\|f^{s}\right\|_{p}\right)<\varepsilon_{0}$.

In the above expressions $f$ and $g$ have the meaning of Lemma (II.1) and $G^{\#}$ as above. The constants $C$ depend on $\varepsilon_{0}$, which is small and fixed.

Proof. The bilinear form $B(u, v)$ admits the domination

$$
\begin{aligned}
|B(u, v)| \leq C \int_{R^{n}}|x-y|^{-n+1} \int_{0}^{t} \mid x- & \left.y\right|^{-2}\left(1+|x-y|^{-1} s^{1 / 2}\right)^{-n-1} \\
& \times|u(y, t-s)||v(y, t-s)| d s d y .
\end{aligned}
$$

The above domination is a consequence of the estimate

$$
\left|D_{k} E_{i j}(x, t)\right| \leq C\left(|x|+t^{1 / 2}\right)^{-n-1} .
$$

Calling $M(u)$ the maximal function of Hardy-Littlewood of $|u|$ on the space variables and $u^{*}$ the sup on $t>0$ of $|u|$, we have

$$
\left|B\left(u, u^{\#}\right)\right| \leq C_{0} \int_{R^{n}}|x-y|^{-n+1}\left(M\left(u^{*}\right)\right)^{2} d y .
$$

The constant $C_{0}$ does not exceed

$$
C \int_{0}^{\infty}\left(1+t^{1 / 2}\right)^{-n-1} d t
$$

The right-hand side of (3.12) does not depend on $t$, hence

$$
\left|B\left(u, u^{\#}\right)\right|^{*} \leq C_{0} \int_{R^{n}}|x-y|^{-n+1}\left(M\left(u^{*}\right)\right)^{2} d y .
$$

We apply now the Hardy-Littlewood-Sobolov potential inequality to (3.14) with exponents $1 / q=2 / n-1 / n$ (see $[12$, pp. 119, 120]), and obtain

$$
\left\|B\left(u, u^{\#}\right)^{*}\right\|_{n}(\infty) \leq C\left\{\left\|M\left(u^{*}\right)\right\|_{n}(\infty)\right\}^{2} \leq C^{\prime}\left\{\left\|u^{*}\right\|_{n}(\infty)\right\}^{2} .
$$

On the other hand, $G^{\#}(x, t)$ satisfies

$$
\begin{aligned}
\left\|\left(G^{\#}\right)^{*}\right\|_{n}(\infty) & \leq C\left\|g^{\#}\right\|_{n} \leq C\|g\|_{n} \\
& \leq C s^{1-p / n}\|g\|_{p}^{p / n} \leq C s^{1-p / n}\|f\|_{p}^{p / n} .
\end{aligned}
$$

The above inequality is an easy consequence of the maximal theorem of HardyLittlewood.

Inequalities (3.15) and (3.16) allow us to apply Lemma (II.2) of $\S$ II and obtain the fixed point if the $L^{n}$-norm of $g$ is suitably small. The smallness of the $\|g\|_{n}$ is guaranteed if $s>0$ is chosen conveniently small. Part (i) of the thesis follows from (3.16) and Lemma (II.2). Clearly, the norm we have used above for the application of Lemma (II.2) is $\left\|()^{*}\right\|_{n}(\infty)$. 
In order to get (ii), we reexamine inequality (3.14) and apply the HardyLittlewood-Sobolov inequality with exponents:

$$
1 / p=1 / r-1 / n, \quad 1 / r=1 / p+1 / n .
$$

This gives

$$
\left\|B\left(u, u^{\#}\right)^{*}\right\|_{p}(\infty) \leq C\left\|u^{*}\right\|_{n}(\infty)\left\|u^{*}\right\|_{p}(\infty) .
$$

We now introduce the norm:

$$
\|u\|=\max \left(\left\|u^{*}\right\|_{n}(\infty),\left\|u^{*}\right\|_{p}(\infty)\right) .
$$

Inequalities (3.15) and (3.17) give immediately a similar inequality to (3.15) for the norm (3.18). Likewise, we introduce the same norm for $G^{\#}(x, t)$, which is going to be suitably small if $\left\|f^{s}\right\|_{p}$ and $s^{1-p / n}\|f\|_{p}^{p / n}$ are.

Lemma (II.2) gives a fixed point $u$ for a suitable Banach ball with the norm (3.18). Inequality (2.13) assures estimate (ii). Finally, all that was said for the cases $u+B\left(u, u^{\#}\right)=G^{\#}$ apply to the limit case $u+B(u, u)=G$, since estimates (3.10)-(3.18) are valid in this case. This concludes the proof.

A priori estimates. Let us consider the approximating system (3.4) and look at the equations containing $v$.

Theorem (III.1). Let $g^{\#}(x)$ be chosen with small $L^{p}$-norm, $\|g\|_{p}<\varepsilon$, such that $u$, whose existence is assured by the lemma above, has a norm not exceeding $a_{0}<1$, that is

$$
\left\|u^{*}\right\|_{n}(\infty)<a_{0}<1
$$

As we did before, $h^{\#}(x)$ will denote the divergence free initial values of $v$, $h^{\#}(x)=\alpha_{\varepsilon} * h$. The functions $g^{\#}$ and $h^{\#}$ have the same meaning as in (3.4), (3.5).

Let $v(x, t)$ be a solution of the approximating system (3.4) for the initial data $h^{\#}$. Suppose that $v, \operatorname{grad} v, D_{t} v$ and $\operatorname{grad} u$ are in $L^{2}$ of $S_{T}$. Then, $v(x, t)$ satisfies the following estimates:

$$
V_{2}(t)+2\left(1-C a_{0}\right)\left\{\|\operatorname{grad} v\|_{2,2}\left(S_{t}\right)\right\}^{2} \leq\left(\left\|h^{\#}\right\|_{2}\right)^{2}, \quad 0<t<T .
$$

Proof. Let us multiply the $L\left(v_{i}\right)$ equation of system (3.4) by $v_{i}$, sum over the index $i$ and integrate over $S_{t}$. It is readily seen by using identity (3.2) that

$$
V_{2}(t)+2\left\{\|\operatorname{grad} v\|_{2,2}\left(S_{t}\right)\right\}^{2}-2\left[(\operatorname{grad} v)\left(v^{\#}\right), u\right]=\left\|h^{\#}\right\|^{2} .
$$

Due to the divergence free property of $v$ and $v^{\#}$ we have

$$
\begin{aligned}
& {\left[(\operatorname{grad} v)\left(v^{\#}\right), v\right]=0,} \\
& {\left[(\operatorname{grad} v)\left(u^{\#}\right), v\right]=0 .}
\end{aligned}
$$


A successive application of Hölder's inequality, first in the space variables for the exponents $r, 2, n ; 1 / r+1 / 2+1 / n=1,1 / r=1 / 2-1 / n$ and second in the time variable for the exponents $2,2, \infty$, gives

$$
\begin{aligned}
\left|\left[(\operatorname{grad} v)\left(v^{\#}\right), u\right]\right| & \leq\left\|v^{\#}\right\|_{r, 2}\left(S_{t}\right)\|\operatorname{grad} v\|_{2,2}\left(S_{t}\right)\left\|u^{*}\right\|_{n} \\
& \leq C a_{0}\left\{\|\operatorname{grad} v\|_{2,2}\left(S_{t}\right)\right\}^{2} .
\end{aligned}
$$

In the above expression we have used Sobolov's inequality, namely

$$
\left\|v^{\#}\right\|_{r, 2}\left(S_{t}\right) \leq\|v\|_{r, 2}\left(S_{t}\right) \leq C\|\operatorname{grad} v\|_{2,2}\left(S_{t}\right), \quad 1 / r=1 / 2-1 / n .
$$

This finishes the proof.

Construction of the weak solution $v(x, t)$. We consider now

$$
v+B\left(v, v^{\#}\right)+B\left(v, u^{\#}\right)+B\left(u, v^{\#}\right)=W * h^{\#} \text {. }
$$

We shall use also the notation $H^{\#}(x, t)$ for the right-hand side of (3.25). We seek a solution $v$ for (3.25), such that $\left\|v^{*}\right\|_{2}(T)<\infty$, for $T$ suitably small.

Lemma (III.2). If $T$ is suitably small, then, there is a solution $v$ to (3.25) such that

$$
\left\|v^{*}\right\|_{2}(T)<\infty
$$

Proof. On account of the definition of $v^{\#}$ we have

$$
\begin{aligned}
\left|B\left(v, v^{\#}\right)\right| & \leq \int_{0}^{t} \int_{R^{n}} K(x-y, t-s) v^{*}(y) \varepsilon^{-n / 2}\left\|v^{*}\right\|_{2}(T) d s d y \\
& \leq C \varepsilon^{-n / 2}\left\|v^{*}\right\|_{2}(T) T^{1 / 2} M\left(v^{*}\right) .
\end{aligned}
$$

Here, $K=C\left(|y|+s^{1 / 2}\right)^{-n-1}$, and $M()$ stands for the $n$-dimensional HardyLittlewood maximal function (with respect to cubes or spheres).

A similar argument gives

$$
\begin{aligned}
\left|B\left(v, u^{\#}\right)\right| & \leq \int_{R^{n}} \int_{0}^{t} K(x-y, t-s) v^{*}(y) M\left(u^{*}\right)(y) d s d y \\
& \leq \int C|x-y|^{-n+1} v^{*}(y) M\left(u^{*}\right) d y .
\end{aligned}
$$

Likewise, we obtain

$$
\left|B\left(u, v^{\#}\right)\right| \leq \int C|x-y|^{-n+1} u^{*}(y) M\left(v^{*}\right) d y .
$$

Inequalities (3.27), (3.28) and (3.29) give immediately

$$
\begin{aligned}
& \left\|\left\{B\left(v, v^{\#}\right)+B\left(v, u^{\#}\right)+B\left(u, v^{\#}\right)\right\}^{*}\right\|_{2}(T) \\
& \quad \leq C\left(\varepsilon^{-n / 2} T^{1 / 2}\left\|v^{*}\right\|_{2}(T)+2\left\|u^{*}\right\|_{n}(\infty)\right)\left\|v^{*}\right\|_{2}(T) .
\end{aligned}
$$

If $\varepsilon^{-n / 2} T^{1 / 2}$ and $\left\|u^{*}\right\|_{n}(\infty)$ are small we can apply Lemma (II.2) and assure the existence of $v$ for small $T$. This finishes the proof.

Remark. Occasionally we write $T$ for $S_{T}$ in the norms involving ( ) ${ }^{*}$ as done in (3.30). 
Lemma (III.3). Let $u(x, t)$ be the solution constructed in Lemma (III.1) solving

$$
u+B\left(u, u^{\#}\right)=G^{\#} .
$$

Let us denote by $D^{\alpha}, \alpha=\left(\alpha_{1}, \ldots, \alpha_{n}\right),|\alpha|=\sum_{j} \alpha_{j}$, the corresponding partial derivative with respect to space variables, and $D_{t}$ the partial derivative with respect to the time. Then, for all $T>0$ and all $\alpha, 0 \leq|\alpha|$, we have

(i) $\left\|D^{\alpha} u\right\|_{n}\left(S_{T}\right)<\infty$,

(ii) $\left\|D_{t} D^{\alpha} u\right\|_{n}\left(S_{T}\right)<\infty$.

Proof. Given the definition of $G^{\#}=W * g^{\#}$, one sees immediately

$$
\sup _{t>0}\left|D^{\alpha} G^{\#}\right| \in L^{n} \quad \text { for all } \alpha .
$$

On the other hand, differentiation of (3.31) gives

$$
D_{i} u+\int_{0}^{t} \int_{R^{n}}\left\langle\left(D_{i} \operatorname{grad} E(x-y, t-s)\right)(u(y, s)), u^{\#}(y, s)\right\rangle d s d y=D_{i} G^{\#}
$$

$D_{i} \operatorname{grad} E$ is the kernel of a convolution singular integral of parabolic type acting on functions of $R^{n+1}$. From the very definition of $u^{\#}$, Hölder's inequality gives

$$
\left\|u^{\#}\right\|_{\infty} \leq C \varepsilon^{-1}\left\|u^{*}\right\|_{n}(\infty) .
$$

In the expression above we have made use of the fact that the function $u^{\#}$ equals $\alpha_{\varepsilon} * u$. The continuity of the convolution singular integral of (3.33) in $L^{n}$ together with (3.34) and (3.32) give the desired property for $D_{i} u$.

The general case for $D^{\alpha} u$ is going to be argued by induction. Suppose that the result holds for $\alpha,|\alpha| \leq k$. Consider $D_{i} D^{\alpha}$ and express (3.31) in terms of convolution on the space and time variable with $W(x, t)$. Let us write also $D^{\alpha}$ as the iterated derivatives $D^{\alpha}=D_{j} D^{\tau}$, where $|\alpha|=k$ and $|\tau|=k-1$. The equation reads

$$
D_{i} D^{\alpha} u+D_{i j} W * \sum C_{\delta \beta} D^{\delta} \operatorname{grad} u D^{\beta} u^{\#}=D_{i} D^{\alpha} G^{\#}, \quad|\delta+\beta|=k-1 .
$$

$D_{i j} W$ is the kernel of a parabolic singular integral operator of convolution type. It should be noted that

$$
\left\|D^{\beta} u^{\#}\right\|_{\infty} \leq\left\|D^{\beta} \alpha_{\varepsilon}\right\|_{n /(n-1)}\left\|u^{*}\right\|_{n}(\infty),
$$

which gives the desired result.

The corresponding result for $D_{t} D^{\alpha}$ follows from two facts:

Fact 1.

$$
D_{t} G^{\#}=\sum D_{i i} G^{\#}
$$

Fact 2.

$$
D_{t} D^{\alpha} u+D_{t} W * D^{\alpha}\left\{(\operatorname{grad} u)\left(u^{\#}\right)\right\}=D_{t} D^{\alpha} G^{\#}
$$


As before, $D_{t} W$ is the kernel of a parabolic singular integral operator. This finishes the proof.

Lemma (III.4). Let $T$ be a value of the time $t$ for which the solution $v$ of (3.39) below is obtainable through the fixed point argument of Lemma (III.2) applied to (3.39):

$$
v+B\left(v, v^{\#}\right)+B\left(v, u^{\#}\right)+B\left(u, v^{\#}\right)=F(x, t) .
$$

In addition, we shall assume that the function $F(x, t)$ satisfies in $S_{T}$

$$
\left\|D^{\alpha} F\right\|_{2}\left(S_{T}\right)<\infty ; \quad\left\|D_{t} D^{\alpha} F\right\|_{2}\left(S_{T}\right)<\infty ; \quad 0 \leq|\alpha| .
$$

In (3.39), we naturally mean the solution u constructed in Lemma (III.1). Then, $v$ satisfies

$$
\begin{aligned}
\left\|D^{\alpha} v\right\|_{2}\left(S_{T}\right) & <\infty ; & 0 \leq|\alpha|, \\
\left\|D_{t} D^{\alpha} v\right\|_{2}\left(S_{T}\right)<\infty ; & & 0 \leq|\alpha| .
\end{aligned}
$$

Proof. As in the previous lemma, we will prove the result by induction. The case $|\alpha|=1$ is concerned with the derivatives $D_{i} v$. Consider the derivative $D_{i}$ of equation (3.39):

$$
D_{i} v+D_{i} B\left(v, v^{\#}\right)+D_{i} B\left(v, u^{\#}\right)+D_{i} B\left(u, v^{\#}\right)=D_{i} F .
$$

In the above expressions for $B$ we use the divergence free fundamental solution $E(x, t)$ as arising in Oseen's formula. We let the kernel $\operatorname{grad} E$ absorb the differentiation in the $B$ 's thus producing a parabolic singular integral transformation of sum of products $v_{i}\left(v_{j}\right)^{\#}, v_{i}\left(u_{j}\right)^{\#}, u_{i}\left(v_{j}\right)^{\#}$ over $S_{t}$.

In (3.43) we show that the above products are in $L^{2}\left(S_{T}\right)$, thus implying the same property for the corresponding $D_{i} B$ 's on account of the continuity in $L^{2}\left(S_{T}\right)$ of the singular integral transformations.

$$
\begin{gathered}
\left\|v_{i}\left(v_{j}\right)^{\#}\right\|_{2}\left(S_{T}\right) \leq\left\|v^{\#}\right\|_{\infty}\|v\|_{2}\left(S_{T}\right) \leq C \varepsilon^{-n / 2}\left\{\|v\|_{2}\left(S_{T}\right)\right\}^{2}, \\
\left\|v_{i}\left(u_{j}\right)^{\#}\right\|_{2}\left(S_{T}\right) \leq\left\|u^{\#}\right\|_{\infty}\|v\|_{2}\left(S_{T}\right), \\
\left\|u_{i}\left(v_{j}\right)^{\#}\right\|_{2}\left(S_{T}\right) \leq\|u\|_{n}\left(S_{T}\right)\left\|v^{\#}\right\|_{q}\left(S_{T}\right) \leq C(\varepsilon, T)\|u\|_{n}\left(S_{T}\right)\|v\|_{2}\left(S_{T}\right), \\
1 / q=1 / 2-1 / n .
\end{gathered}
$$

Consider now $D_{i} D^{\alpha}$, where $|\alpha|=k-1$ and let us assume the property valid for all $\delta$ such that $|\delta| \leq k-1$. We write

$$
\begin{aligned}
D_{i} D^{\alpha} & +\sum C_{\delta \beta} D_{i} B\left(D^{\delta} v, D^{\beta} v^{\#}\right)+\sum C_{\delta \beta} D_{i} B\left(D^{\delta} v, D^{\beta} u^{\#}\right) \\
& +\sum C_{\delta \beta} D_{i} B\left(D^{\delta} u, D^{\beta} v^{\#}\right)=D_{i} D^{\alpha} F ; \quad|\delta+\beta| \leq k-1 .
\end{aligned}
$$

The expression above is a consequence of Leibnitz's formula. The continuity in $L^{2}\left(S_{T}\right)$ of the parabolic singular integral operators in $(3.44)$ give the desired 
property on account of estimates (3.45):

$$
\begin{gathered}
\left\|D^{\delta} v\right\|_{2}\left(S_{T}\right)<\infty ; \quad\left\|D^{\beta} v^{\#}\right\|_{\infty}\left(S_{T}\right)<\infty, \\
\left\|D^{\beta} u^{\#}\right\|_{\infty}\left(S_{T}\right)<\infty ; \quad\left\|D^{\delta} u\right\|_{n}\left(S_{T}\right)<\infty, \\
\left\|D^{\beta} v^{\#}\right\|_{q}\left(S_{T}\right) \leq C(\varepsilon, T)\left\|D^{\beta} v\right\|_{2}\left(S_{T}\right) ; \quad 1 / q=1 / 2-1 / n .
\end{gathered}
$$

In (3.45) above we have $|\delta+\beta| \leq k-1$. This proves (i). The proof of (ii) follows the same lines but it uses $W$ instead of $E$. We are interested in estimating $D_{t} D^{\alpha} v$, hence we use

$$
\begin{aligned}
D_{t} D^{\alpha} v & +D_{t} W * D^{\alpha}\left\{(\operatorname{grad} v)\left(v^{\#}\right)\right\}+D_{T} W * D^{\alpha}\left\{(\operatorname{grad} v)\left(u^{\#}\right)\right\} \\
& +D_{t} W * D^{\alpha}\left\{(\operatorname{grad} u)\left(v^{\#}\right)\right\}=D_{t} D^{\alpha} F .
\end{aligned}
$$

On account of (i) and the fact that $D_{t} W$ is the kernel of a parabolic singular integral operator (ii) is established.

Theorem (III.3). The solution constructed in Lemma (III.2) can be extended to all time $t>0$, and furthermore it satisfies the "a priori estimates" of Theorem (III.2) for all $t>0$, that is

$$
\begin{aligned}
& \frac{1}{2} \int_{R^{n}}|v(x, t)|^{2} d x+\left(1-a_{0}\right) \int_{0}^{t} \int_{R^{n}}|\operatorname{grad} v|^{2} d x d t \\
& \leq \frac{1}{2} \int_{R^{n}} \mid h^{\#} \|^{2} d x .
\end{aligned}
$$

Proof. We start with the initial values $h^{\#}(x)$ and construct a solution $v(x, t)$ by using the fixed point argument of Lemmae (II.2) and (III.2) for small $T$. The size of $T$ depends on $C \varepsilon^{-n / 2} T^{1 / 2}, C\left\|u^{*}\right\|_{n}(\infty)$ and $C\left\|h^{\#}\right\|_{2}$, here $C$ is an independent constant. It should be clear that instead of $\left\|u^{*}\right\|_{n}(\infty)$ we can use $\left\|u^{*}\right\|_{n}(T)$ as well. Invoking Lemma (II.2) once more, we see that the condition that gives the size of $T$ is

$$
\begin{aligned}
& 2 C \varepsilon^{-n / 2} T^{1 / 2}\left\|h^{\#}\right\|_{2}\left\{\left(1-C\left\|u^{*}\right\|_{n}\right)^{2}-4 C \varepsilon^{-n / 2} T^{1 / 2}\left\|h^{\#}\right\|_{2}\right\}^{-1 / 2} \\
& \quad+C\left\|u^{*}\right\|_{n}(\infty)<1 .
\end{aligned}
$$

Here, as before $C$ is an independent constant.

The regularity Lemma (III.4) allows us to apply the "a priori estimates" of Theorem (III.2) and move from $t=0$ to $t=T$ without increasing the $L^{2}$-norm of the initial data. As a byproduct of Lemma (III.4) we obtain that $D^{\alpha} v(x, T)$ is in $L^{2}$ for all $\alpha$; and furthermore, we have

$$
D^{\alpha} v(x, t)=\int_{0}^{t} D_{s} D^{\alpha} v(x, s) d s+D^{\alpha} h^{\#}(x),
$$

and consequently

$$
\left\{\int_{R^{n}}\left|D^{\alpha} v(x, T)\right|^{2} d x\right\}^{1 / 2} \leq\left\|D_{t} D^{\alpha} v\right\|_{2}\left(S_{T}\right)+\left\|D^{\alpha} h^{\#}\right\|_{2} .
$$


The strategy is now clear, we move from 0 to $T$, and from $T$ to $2 T$ and so on without increasing the $L^{2}$-norm of the initial data and being assured all the regularity we need to apply the "a priori estimates" on each step. This finishes the proof.

\section{A refinement of the "a priori estimates".}

Theorem (III.4). Let $\beta(x)$ be a nonnegative $C^{\infty}$-infinity function such that $\beta=$ 1 if $|x|>N$ and $\beta=0$ if $|x|<N / 2,|\operatorname{grad} \beta| \leq C / N, n=3,4$. The function $v$ (solution of (3.4) for the initial data $h^{\#}$ known to exist for all time $t$ by virtue of the preceding results) satisfies the following estimate:

$$
\begin{aligned}
\frac{1}{2} \int_{R^{n}} \beta(x)|v(x, t)|^{2} d x+\iint_{S_{t}} \beta|\operatorname{grad} v|^{2} d x d s & \\
\leq & \frac{1}{2}\left(\left\|\beta h^{\#}\right\|_{2}\right)^{2}+C\left(\frac{1+t}{N}\right)\left(\left\|h^{\#}\right\|_{2}\right)^{2} \\
& +(C / N)\|u\|_{n, \infty}(T)\left(\left\|h^{\#}\right\|_{2}\right)^{2}+C\|\beta u\|_{n, \infty}(T)\left(\left\|h^{\#}\right\|_{2}\right)^{2}, \\
& 0<t<T .
\end{aligned}
$$

In (3.51) above, $C$ stands for an independent constant and $u$ is the solution constructed in Lemma (III.1).

Proof. Let us return to the portion of the approximating system (3.4) involving $v$, namely

$$
L(v)+(\operatorname{grad} v)\left(v^{\#}\right)+(\operatorname{grad} v)\left(u^{\#}\right)+(\operatorname{grad} u)\left(v^{\#}\right)+\operatorname{grad} Q=0 .
$$

The pseudopressure $Q$ is given by

$$
Q=C \sum R_{i} R_{j}\left(v_{i}\left(v_{j}\right)^{\#}+v_{i}\left(u_{j}\right)^{\#}+u_{i}\left(v_{j}\right)^{\#}\right) .
$$

Here, $R_{i}$ denotes as usual the $i$ th Riesz transform. Let us multiply the $i$ th equation of (3.52) by $\beta v_{i}$, sum over $i$ and integrate over $S_{t}$, namely

$$
\begin{gathered}
\frac{1}{2} \int_{R^{n}} \beta|v(x, t)|^{2} d x+\iint_{S_{t}} \beta|\operatorname{grad} v|^{2} d x d s+[(\operatorname{grad} v)(\operatorname{grad} \beta), v] \\
+\left[(\operatorname{grad} v)\left(v^{\#}\right), \beta v\right]+\left[(\operatorname{grad} u)\left(v^{\#}\right), \beta v\right]+\left[(\operatorname{grad} v)\left(u^{\#}\right), \beta v\right] \\
\quad-\iint_{S_{t}}\langle\operatorname{grad} \beta, v\rangle Q d x d s=\frac{1}{2} \int_{R^{n}} \beta\left|h^{\#}\right|^{2} d x .
\end{gathered}
$$

We are going to deal separately with the $3 \mathrm{rd}, 4 \mathrm{th}, 5 \mathrm{th}, 6$ th and 7 th terms of the left-hand side in (3.54) above.

3 rd term.

$$
|[(\operatorname{grad} v)(\operatorname{grad} \beta), v]| \leq(C / N)\|\operatorname{grad} v\|_{2}\left(S_{t}\right)\|v\|_{2}\left(S_{t}\right) .
$$

4th term.

$$
\left[(\operatorname{grad} v)\left(v^{\#}\right), \beta v\right]=-\left[(v \operatorname{grad} \beta)\left(v^{\#}\right), v\right]-\left[\beta(\operatorname{grad} v)\left(v^{\#}\right), v\right] .
$$


Hence

$$
\left[(\operatorname{grad} v)\left(v^{\#}\right), \beta v\right]=-\frac{1}{2}\left[(v \operatorname{grad} \beta)\left(v^{\#}\right), v\right] .
$$

An application of Hölder's inequality to the right-hand side of (3.56) gives

$$
\left|\left[(\operatorname{grad} v)\left(v^{\#}\right), \beta\right]\right| \leq \frac{C}{N} \int_{0}^{t}\left(\int_{R^{n}}|v(x, s)|^{2} d x \int_{R^{n}}|v|^{2}\left|v^{\#}\right|^{2} d x\right)^{1 / 2} d s .
$$

Denoting by $M()$ the Hardy-Littlewood maximal function with respect to spheres in the space variables we obtain the following domination for the functions of (3.57):

$$
\int_{R^{n}}|v|^{2}\left|v^{\#}\right|^{2} d x \leq \int_{R^{n}} M^{4}(v) d x \leq C \int_{R^{n}}|v|^{4} d x .
$$

Since $n=3,4$, we have

$$
\int_{R^{n}}|v|^{4} d x \leq C\left(\int_{R^{n}}\left\{|\operatorname{grad} v|^{2}+|v|^{2}\right\} d x\right)^{2} .
$$

The above inequality is an easy consequence of Sobolov's inequality.

Now we can use (3.58) and (3.59) to dominate the right-hand side of (3.57) and obtain

$$
\left|\left[(\operatorname{grad} v)\left(v^{\#}\right), \beta v\right]\right| \leq C / N\left(\left\|h^{\#}\right\|_{2}+(1+t)\left(\left\|h^{\#}\right\|_{2}\right)^{2}\right) .
$$

5 th term. For the 5 th term we have

$$
\left[(\operatorname{grad} u)\left(v^{\#}\right), \beta v\right]=-\left[(v \operatorname{grad} \beta)\left(v^{\#}\right), u\right]-\left[\beta(\operatorname{grad} v)\left(v^{\#}\right), u\right],
$$

which gives immediately

$$
\begin{aligned}
\left|\left[(v \operatorname{grad} \beta)\left(v^{\#}\right), u\right]\right| & \leq(C / N)\|u\|_{n, \infty}(T) \iint_{S_{l}}|\operatorname{grad} v|^{2} d x d s \\
& \leq(C / N)\|u\|_{n, \infty}(T)\left(\left\|h^{\#}\right\|_{2}\right)^{2}, \quad 0<t<T
\end{aligned}
$$

A similar argument gives

$$
\left|\left[\beta(\operatorname{grad} v)\left(v^{\#}\right), u\right]\right| \leq C\|u \beta\|_{n, \infty}(T)\left(\left\|h^{\#}\right\|_{2}\right)^{2}, \quad 0<t<T .
$$

6th term. We use here the same argument as in previous case:

$$
\begin{aligned}
\left|\left[(\operatorname{grad} v)\left(u^{\#}\right), \beta v\right]\right| & \leq\|\beta u\|_{n, \infty}(T) \iint_{S_{t}}|\operatorname{grad} v|^{2} d x d s \\
& \leq\|\beta u\|_{n, \infty}(T)\left(\left\|h^{\#}\right\|_{2}\right)^{2}, \quad 0<t<T .
\end{aligned}
$$

7th term, the pseudopressure $Q$. On account of the expression we have for $Q$ in terms of singular integral transformations (linear combinations of composition of Riesz operators), which generically will be designated by the symbol $K()$, we have to deal with integrals of the type

$$
\begin{aligned}
& \text { (a) } \iint_{S_{t}}\langle\operatorname{grad} \beta, v\rangle K\left(v_{i}\left(v_{j}\right)^{\#}\right) d x d s, \\
& \text { (b) } \iint_{S_{t}}\langle\operatorname{grad} \beta, v\rangle K\left(v_{i}\left(u_{j}\right)^{\#}\right) d x d s, \\
& \text { (c) } \iint_{S_{t}}\langle\operatorname{grad} \beta, v\rangle K\left(u_{i}\left(v_{j}\right)^{\#}\right) d x d s .
\end{aligned}
$$


The fact that $K$ defines a bounded mapping from $L^{q}\left(R^{n}\right)$ into itself, $1<q<$ $\infty$, allows us to treat (a) as the 4 th term above, and (b), (c), as the 5 th term. Estimates (3.55), (3.60), (3.62), (3.63), (3.64) and what was said about the 7 th term conclude the proof.

End of the proof of Theorem A, compactness arguments.

Lemma $\mathbf{A}_{1}$. (i) There is a $T>0$ depending on the following norm of $g$ :

$$
\|g\|=\|g\|_{n}+\|g\|_{n / \beta}, \quad 0<\beta<1,
$$

such that the family of functions

$$
u(x, t)
$$

solutions of $u+B\left(u, u^{\#}\right)=G^{\#}$ as a family dependent on the parameters $\varepsilon$ and $t, 0<t<T$ is compact in $L^{n}$.

(ii) The size of $T$ is determined by the condition

$$
T^{(1-\beta) / 2}\left(s^{1-\beta p / n}\|f\|_{p}^{\beta p / n}+s^{1-p / n}\|f\|_{p}^{p / n}\right)<\varepsilon_{0} .
$$

Furthermore, we have for the solutions the estimates

(iii)

$$
\begin{gathered}
\left\|u^{*}\right\|_{n}(T) \leq C\left(\varepsilon_{0}\right)\left(s^{1-\beta p / n}\|f\|_{p}^{\beta p / n}+s^{1-p / n}\|f\|_{p}^{p / n}\right) \\
\left\|u^{*}\right\|_{p}(T) \leq C^{\prime}\left(\varepsilon_{0}\right)\left(s^{1-\beta p / n}\|f\|_{p}^{\beta p / n}+\left\|f^{s}\right\|_{p}\right)
\end{gathered}
$$

Here, the $u(x, t)$ are solutions of (3.4) for the initial values $g^{\#}$. The constants $C$ and $C^{\prime}$ depend on $\varepsilon_{0}$, which is a fixed small quantity arising from (2.11), (2.12) and (2.13) in Lemma (II.2).

Proof. Consider the following estimate for $E$ :

$$
|E| \leq C|x|^{\beta-n}|t|^{-(1+\beta) / 2}, \quad 0<\beta<1,
$$

which gives for the transformation $m=B\left(u, w^{\#}\right)$ the estimates

$$
\begin{aligned}
\left\|m^{*}\right\|_{n / \beta}(T) & \leq C T^{(1-\beta) / 2}\left\|u^{*}\right\|_{n / \beta}(T)\left\|\left(w^{\#}\right)^{*}\right\|_{n / \beta}(T), \\
\left\|m^{*}\right\|_{n}(T) & \leq C T^{(1-\beta) / 2}\left\|u^{*}\right\|_{n}(T)\left\|\left(w^{\#}\right)^{*}\right\|_{n / \beta}(T),
\end{aligned}
$$

that are easy consequences of Sobolov's potential inequality. The combination of both estimates in (3.69) above gives us

$$
\|m\| \leq C T^{(1-\beta) / 2}\|u\| \cdot\left\|w^{\#}\right\|,
$$

where the \|\| norm is the sum of the $n$ and $n / \beta$ norms of (3.69). An application of the fixed point Lemma (II.2) to

$$
u+B\left(u, u^{\#}\right)=G^{\#}
$$


using the (3.70) norm, gives $u$ with $u_{T}^{*}$ in the intersection of $L^{n}$ and $L^{n / \beta}$. Hence

$$
\begin{aligned}
\left\|\left(u-G^{\#}\right)^{*}\right\|_{n}(T) & \leq C T^{(1-\beta) / 2}\left\|u^{*}\right\|_{n}(T)\left\|\left(u^{\#}\right)^{*}\right\|_{n / \beta}(T) \\
& \leq C^{\prime} T^{(1-\beta) / 2} M .
\end{aligned}
$$

Here, $M$ is any bound for corresponding norms of the fixed point in terms of the initial data from Lemma (II.2). The inequalities (3.72) specialized to subintervals of $(0, T)$, namely

$$
\left\|\left(u\left(x, t_{0}+t\right)-W * u\left(x, t_{0}\right)\right)^{*}\right\|_{n}(t)<C^{\prime} M t^{(1-\beta) / 2}
$$

together with the compactness of $G^{\#}$ complete the proof of (i). Parts (ii) and (iii) follow from an application of Lemma (II.2) to $u+B\left(u, u^{\#}\right)=G^{\#}$ using the norms

$$
\left.\left\|()^{*}\right\|_{n / \beta}(T)+\left\|()^{*}\right\|\right)_{n}(T), \quad\left\|()^{*}\right\|_{n / \beta}(T)+\left\|()^{*}\right\|_{p}(T),
$$

and (3.69) together with a similar estimate to (3.69), namely

$$
\left\|m^{*}\right\|_{p}(T) \leq C T^{(1-\beta) / 2}\left\|u^{*}\right\|_{p}(T)\left\|\left(w^{\#}\right)^{*}\right\|_{n / \beta}(T)
$$

which is a simple consequence of (3.68) and Sobolov's potential inequality. This concludes the proof of Lemma $A_{1}$.

Observation. Lemma $\mathrm{A}_{1}$ implies that by selecting $N$ large enough (the one associated with $\beta(x))$ the term in (3.51) involving $\|(\beta u)\|_{n, \infty}(T)$ can be made uniformly small independently from $\varepsilon$.

Lemma $\mathbf{A}_{2}$. The family of solutions $v$ of (3.4) for the initial values $h^{\#}$, as a family depending on the parameter $\varepsilon$, contains a denumerable subfamily that converges in $L^{2}$ of any subset $S_{T}, n=3,4, T>0$.

Proof. Consider the functions $V(t)$ defined by

$$
V^{2}(t)=\int_{R^{n}}|v(x, t)|^{2} d x
$$

Let us bring back Theorem (III.2) and write the basic identity

$$
\left[(\operatorname{grad} v)\left(v^{\#}\right), u\right]+\left(\|\operatorname{grad} v\|_{2}\left(S_{t}\right)\right)^{2}+(1 / 2) V^{2}(t)=(1 / 2)\left(\left\|h^{\#}\right\|\right)^{2} .
$$

The "a priori estimates" give the fact that the first and second term in the left-hand side above have uniformly bounded variation as functions of $t$; see Theorem (III.1) and inequality (3.23) in its proof. The identity (3.74) above implies the same property for $V^{2}(t)$. Helly's selection principle allows us to extract a sequence of $V^{2}(t) s$ converging to a limit $X(t)$ except for a set at most countable. Likewise, Alaoglu's theorem allows us to extract a sequence of $v, s$ converging together with their space derivatives weakly in $L^{2}\left(S_{T}\right)$ to a limit function $v$.

J. Leray's argument [8, paragraphs 29 and 30, pp. 236-240] adapted to this case thanks to Theorem (III.4) and Lemma $A_{1}$ gives the strong convergence in 
$L^{2}$ of the sequence of $v, s$. For the sake of completeness let us illustrate the heart of the argument.

Call $v_{m}$ the selected sequence converging together with the sequences of space variable derivatives to $v$ in the weak topology of $L^{2}\left(S_{T}\right)$, assume also that for the same sequence $\left(V_{m}(t)\right)^{2}$ is convergent except for a countable set. From the "a priori estimates" we get

$$
\int_{R^{n}}\left|\operatorname{grad} v_{m}\right|^{2} d x \leq A(t)<\infty \quad \text { a.e. }
$$

Inequality (3.75) implies that there is an appropriate subsequence of $v_{m}(x, t)$ depending on $t$, that converges to $v$ in $L^{2}$ of bounded measurable subsets of $R^{n}$. The estimates that we have on the size of the tails, from Lemma $A_{1}$ and Theorem (III.4), give the inequality below by selecting $N$ large enough:

$$
\int_{|x|>N}|v|^{2} d x<\delta .
$$

The estimate above implies the convergence of $v_{m(t)}$ to $v^{\sim}$ in $L^{2}\left(R^{n}\right)$. From this point one sees that

$$
\left(V_{m(t)}\right)^{2} \text { converges to } \int_{R^{n}}\left|v^{\sim}\right|^{2} d x .
$$

Integration with respect to $t$ shows that $\left\|v_{m}\right\|_{2}\left(S_{T}\right)$ tends to $\left\|v^{\sim}\right\|_{2}\left(S_{T}\right)$ and a well-known result of $\mathrm{F}$. Riesz finishes the argument.

Final steps. As a consequence of Lemmas $A_{1}$ and $A_{2}$ we construct a sequence of solutions $v_{m}$ and $u_{m}$ to the approximating systems (3.4), such that both sequences converge in $L^{2}\left(S_{T}\right)$ and $L^{n}\left(S_{T}\right)$ respectively.

Let us take now a test function $w(x, t)$ [see Ia) and $\mathrm{b}$ )], integrate against equations (3.4) specialized on $v_{m}$ and $u_{m}$, use the identities (3.2) and (3.3), and let $m$ go to $\infty$. We obtain solutions of system (2.6) by virtue of the facts:

(a) Products of components of $v_{m}$ and $v_{m} * \alpha_{m}$ converge in $L^{2}\left(S_{T}\right)$.

(b) Products of components of $v_{m}$ and $u_{m} * \alpha_{m}$, or conversely, converge in $L^{q}\left(S_{T}\right), 1 / q=1 / 2+1 / n$.

(c) Products of components of $u_{m}$ and $u_{m} * \alpha_{m}$ converge in $L^{n / 2}\left(S_{T}\right)$.

(d) $h_{m}$ and $g_{m}$ converge to $h$ and $g$ in $L^{2}$ and $L^{n}$ respectively.

In order to show part (i) of Theorem A consider the sequence $u_{m}$ known to converge in $L^{n}\left(S_{T}\right)$ for a suitable $T>0$ (this is a consequence of Lemma $\mathrm{A}_{1}$ ), we may assume as well that the sequence converges a.e. to $u$. This fact implies that the limit function $u$ satisfies

$$
u+B(u, u)=G .
$$

An application of Lemma $\mathrm{A}_{1}$ to $u_{m}$ gives

$$
\left\|\left(u_{m}\right)^{*}\right\|_{p}(T) \leq C\left(\varepsilon_{0}\right)\left(s^{1-\beta p / n}\|f\|_{p}^{\beta p / n}+\left\|f^{s}\right\|_{p}\right)
$$


where $T$ is restricted as in Lemma $\mathrm{A}_{1}$. The passage to the norm \|\|$_{p, 2}\left(S_{T}\right)$ introduces the extra factor $T^{1 / 2}$ on the right-hand side of (3.79). Fatou's theorem gives

$$
\|u\|_{p, 2}(T) \leq C\left(\varepsilon_{0}\right) T^{1 / 2}\left(s^{1-\beta p / n}\|f\|_{p}^{\beta p / n}+\left\|f^{s}\right\|_{p}\right) .
$$

Now, we proceed to study $v_{m}$, which is assumed to converge in norm $L^{2,2}\left(S_{T}\right)$ and also a.e. From previous results and Sobolov's imbedding theorem we have

$$
\begin{aligned}
\left\|v_{m}\right\|_{p, 2}(T) & \leq C\left(\left\|\operatorname{grad} v_{m}\right\|_{2,2}(T)+\left(V_{m}\right)_{2}(T)\right) \\
& \leq C^{\prime}\left\|h^{\#}\right\|_{2} \leq C^{\prime \prime} s^{1-p / 2}\|f\|_{p}^{p / 2} .
\end{aligned}
$$

Fatou's theorem gives

$$
\|v\|_{p, 2}(T) \leq C^{\prime \prime} s^{1-p / 2}\|f\|_{p}^{p / 2} .
$$

Inequalities (3.80) and (3.82) give part (i) of the thesis.

In order to prove (ii), that is to extend the solution $u+v$ constructed in the previous step to all time $t>T$, we observe that $u$ has finite $L^{p}$ norm on the space variables for all $t$, such that $0 \leq t \leq T$, likewise $v$ has finite $L^{2}$ norm on the space variables for all $t, 0 \leq t \leq T$. We partition $u$ for $t=T$ as the sum $u^{\prime}+u^{\prime \prime}$ in the same fashion as in Lemma (II.1) and go about the same construction used to prove part (i) with the following definitions for $h$ and $g$ :

$$
h_{1}=u^{\prime \prime}+v(x, T), \quad g_{1}=u^{\prime} .
$$

The selection of $u^{\prime}$ with small $L^{p}$ norm is going to assure the existence of a solution $u_{1}+v_{1}$ for all time $t, T \leq t \leq T_{1}$. Finally, observing that the $L^{p, 2}$ norm of $v_{1}$ over $R^{n} \times\left(T, T_{1}\right)$ depends only on the $L^{2}$ norms $(0,4)$, $T \leq t \leq T_{1}$, of $v_{1}$ and the $\operatorname{grad} v_{1}$ over $R^{n} \times\left(T, T_{1}\right)$, we obtain (ii) for $t$ within the range $T \leq t \leq T_{1}$.

The previous step illustrates the "basic step" for the induction. This is accomplished by repeating the basic step in each portion $\left(T_{k}, T_{k+1}\right) \times R^{n}$. In each step we select $g_{k}$ with $L^{p}$ norm suitably small to assure $T_{k+1}-T_{k}>\delta_{0}$ for all $k$. Details are left to the reader.

Results for the limiting case $p=n$. It follows from the previous theory that the partitions and techniques covering the cases $2<p<n, 3 \leq n \leq 4$, apply also to this case. In this situation however, it is possible to obtain results that are qualitatively better and of slightly different nature. As we are going to see, it will be possible to imbed the $L^{n}$ initial data in the existence and uniqueness class $L^{n+2, n+2}\left(S_{T}\right)$, treated by Fabes, Jones and Riviere in [3, Theorems (3.2) and (3.3)].

Theorem B. Suppose that $f(x)$, the initial data, is a vector function, such that $f \in L^{n}\left(R^{n}\right), \operatorname{div} f=0$ (in the distributions sense). If $\|f\|_{n}<\varepsilon_{0}, n \geq 3$, there 
is a unique global solution $u$ to problem $(0.1)$ such that

(i) $\left\|u^{*}\right\|_{n}(\infty) \leq C\left(\varepsilon_{0}\right)\|f\|_{n}$.

Here, $\varepsilon_{0}$ is a fixed constant that depends on the fixed point estimates of Lemma (II.2). If no restriction is imposed on the size of $\|f\|_{n}$, then there exists a unique solution $u$ in $L^{n+2, n+2}\left(S_{T}\right), n \geq 2$, provided that

(ii) $\|W * f\|_{n+2, n+2}\left(S_{T}\right) \leq \varepsilon_{0}$.

Moreover, we always have

(iii) $\|W * f\|_{n+2, n+2}(\infty) \leq C(n)\|f\|_{n} \cdot{ }^{1}$

The constant $\varepsilon_{0}$ has the same meaning as above. $W$ denotes as always the Weierstrass kernel and the convolution is on the spatial variables.

Proof. Part (i) follows from Lemma (II.2) and inequalities (3.12)-(3.16) of Lemma (III.1). On account of Theorems (3.1), (3.2) and (3.3) in [3] it will be enough to show only part (iii).

Call $F(x, t)$ the following function:

$$
F(x, t)=t^{-n / 2} \int_{|x-y|<t^{1 / 2}} f(y) d y .
$$

On account of the fact that for $f \geq 0$ we have

$$
W * f \leq \text { constant } \sum_{1}^{\infty} b_{k} 2^{n k} F\left(x, 2^{2 k} t\right),
$$

where $b_{k}=\exp -\left[2^{(k-1)}\right]^{2}$, and of the "a priori estimate" derived from (3.85)

$$
\|W * f\|_{n+2, n+2}(\infty) \leq \mathrm{const} \sum_{1}^{\infty} b_{k} 2^{n k\{1-2 /[p(n+2)]\}}\|F\|_{n+2, n+2}(\infty)
$$

it will be enough to prove (iii) for $F(x, t)$ instead of $W * f$. Without loss of generality, we may assume that $F(x, t)$ refers to "cubes", with sides parallel to the coordinate axes, being centered at $x$ and having edges of length $2 t^{1 / 2}$. On account of the "a priori estimate" (3.86), (iii) will be a consequence of the following imbedding lemma:

Lemma $\mathbf{A}_{3}$. Let $1 \leq n<\infty, f \in L^{p}\left(R^{n}\right), 1<p<\infty$. Then the function $F(x, t)$ defined above belongs to $L^{(n+2) p / n,(n+2) p / n}(\infty)$; furthermore, we have the inequality

(i) $\|F\|_{(n+2) p / n,(n+2) p / n}(\infty) \leq C_{p}(n)\|f\|_{p}$.

The constant $C_{p}(n)$ above depends only on $p$ and on $n$.

Proof. Without loss of generality we may assume that $f \geq 0$ and that $\|f\|_{p}=1$. Consider now $G$ to be the set of points $R^{n}$ where $M(f)>s$. Here $M()$ stands for the maximal function of $f$ with respect to centered cubes with edges parallel to the coordinate axes. The set $G=G(s)$ is an open set, and $\cup Q_{k}$ will denote a cover of it in the Whitney sense (see [12, pp. 167-169]).

\footnotetext{
${ }^{1}$ Parts (ii) and (iii) have been obtained independently by E. B. Fabes and Fred Weissler.
} 
The following properties are easy to verify and constitute standard arguments:

(1) The cubes $Q_{k}$ are nonoverlapping, $\cup Q_{k}=G$.

(2) $c_{1} \operatorname{dist}\left(Q_{k}, B\right) \leq \operatorname{diam}\left(Q_{k}\right) \leq c_{2} \operatorname{dist}\left(Q_{k}, B\right), B=\operatorname{complement}(G)$.

(3) $M(f) \leq s$ if $x \in B$.

(4) If $x \in Q_{k}$ and $Q$ is a cube centered at $x$ and such that $\operatorname{diam}(Q) \leq$ $c_{3} \operatorname{diam}\left(Q_{k}\right)$, then $|Q|^{-1} \int_{Q} f d y \geq c_{4} s$.

In the above expression, $c_{3}$ and $c_{4}$ are universal constants that are larger than 2 . The constant $c_{3}$ is selected so that $Q$ touches $B$. Keeping the same notation, the edges of the cubes are given by the values $2 t^{1 / 2}$ for $t=t_{k}$, $k=1,2,3, \ldots$. The measure of each $\left|Q_{k}\right|$ is dominated by constant $\left(s^{-p}\right)$. We introduce in $R^{n+1}$ the sets

$$
R_{k}=\left\{(x, t) ; x \in Q_{k}, 0<t<\left[c_{3} \operatorname{diam}\left(Q_{k}\right)\right]^{2}\right\} .
$$

By construction, we have

$$
\begin{aligned}
& F \leq s \quad \text { if } x \in B, \\
& F \leq c_{4} s \quad \text { if } x \in Q_{k} \text { and } t>\left[c_{3} \operatorname{diam}\left(Q_{k}\right)\right]^{2} .
\end{aligned}
$$

The above inequalities imply that $\left\{F>\left(c_{4}\right) s\right\}$ is contained in $\bigcup_{1}^{\infty} R_{k}$. We have the following estimate for the above union:

$$
\begin{aligned}
\left|\bigcup R_{k}\right| & \leq \text { constant }\left[\sup \operatorname{diam}\left(Q_{k}\right)\right]^{2} \sum\left|Q_{k}\right| \\
& \leq \text { constant } s^{-2 p / n}|G| \leq \text { constant } s^{-2 p / n} D(s) .
\end{aligned}
$$

In the above expression $D(s)$ stands for the distribution function of $M(f)$ and we have used $\left|Q_{k}\right|<C s^{-p}$. Calling $D_{0}(s)$ the distribution function of $F(x, t)$ over the upper half-space of $R^{n+1}$ we get as a consequence of (3.89)

$$
D_{0}(s) \leq \text { constant } s^{-2 p / n} D\left(c_{4} s\right) \text {. }
$$

Here, $c_{5}$ is the inverse of $2\left(c_{4}\right)$. Finally, integrating $D_{0}(s)$ against $s^{(n+2) p / n-1}$ from 0 to $\infty$ and using (3.90) we obtain the thesis for nonnegative $f,\|f\|_{p}=$ 1 . The general case follows from addition and homogeneity.

Observation. The connection between the solutions of Theorems A and B remains as an open problem.

\section{REFERENCES}

1. A. Benedek and R. Panzone, The space $L^{p}$ with mixed norm, Duke Math. J. 28 (1961), 301-324.

2. E. B. Fabes and N. M. Riviere, Singular integrals with mixed homogeneity, Studia Math. 27 (1966), 19-38.

3. E. B. Fabes, B. F. Jones, and N. M. Riviere, The initial value problem for the Navier-Stokes equations with data in $L^{P}$, Arch. Rational Mech. Anal. 45 (1972), 222-240.

4. E. B. Fabes, J. E. Lewis, and N. M. Riviere, Singular integrals and hydrodynamical potentials, Amer. J. Math. 99 (1977), 601-625. 
5. __ Boundary value problems for the Navier-Stokes equations, Amer. J. Math. 99 (1977), 626-668.

6. E. Hopf, Ueber die Anfangsaufgabe für die hydrodinamischen Grundgleichungen, Math. Nachr. 4 (1951).

7. O. A. Ladyzhenskaja, The mathematical theory of viscous incompressible flow, Gordon and Breach, 1969.

8. J. Leray, Sur le mouvement d'un liquide visqueux emplissant lespace, Acta Math. 63 (1934), 193-248.

9. C. W. Oseen, Neuere Methoden und Ergebnisse in der Hydrodinamik, Akademie Verlagsgessellsschaft, Leipzig, m.b.h. 1927, p. 68.

10. G. Prodi, Un teorema di unicitá per le equazioni di Navier Stokes, Ann. Mat. Pura Appl. 48 (1959), 173-182.

11. J. Serrin, The initial value problem for the Navier Stokes equations, Nonlinear Problems (R. E. Langer, ed.), Univ. of Wisconsin Press, 1963, pp. 69-83.

12. E. M. Stein, Singular integrals and differentiability properties of functions, Princeton Univ. Press, N.J., 1970.

13. F. B. Weissler, Initial value problem in $L^{p}$ for the Navier Stokes fluids, Arch. Rational Mech. Anal. 74 (1980), 219-229.

Department of Mathematics, University of Illinois at Chicago, Chicago, Illinois 60680 